\title{
Background, But Not Foreground, Spatial Cues Are Taken as References for Head Direction Responses by Rat Anterodorsal Thalamus Neurons
}

\author{
Michaël B. Zugaro, Alain Berthoz, and Sidney I. Wiener \\ Centre National de la Recherche Scientifique, Collège de France, Laboratoire de Physiologie de la Perception et de \\ l'Action, 75231 Paris CEDEX 05, France
}

\begin{abstract}
Two populations of limbic neurons are likely neurophysiological substrates for cognitive operations required for spatial orientation and navigation: hippocampal pyramidal cells discharge selectively when the animal is in a certain place (the "firing field") in the environment, whereas head direction cells discharge when the animal orients its head in a specific, "preferred" direction. Cressant et al. (1997) showed that the firing fields of hippocampal place cells reorient relative to a group of three-dimensional objects only if these are at the periphery, but not the center of an enclosed platform. To test for corresponding responses in head direction cells, three objects were equally spaced along the periphery of a circular platform. Preferred directions were measured before and after the group of objects
\end{abstract}

In monkeys, rats, and mice, two types of limbic system neurons have been observed that may function as neurophysiological substrates for spatial orientation. Hippocampal neurons discharge selectively when the animal is at a certain location in the environment (the firing field of the "place" cell; O'Keefe and Conway, 1978; Ono et al., 1991; Rotenberg et al., 1996; McHugh et al., 1996), whereas head direction (HD) cells discharge only when the animal orients its head in a specific direction (the preferred direction of the cell; Ranck, 1984; Taube, 1998; Robertson et al., 1999; Khabbaz et al., 2000). The head direction signal is found in an ascending series of nuclei known as the "Papez circuit", projecting to the hippocampus. A yet unsolved problem concerns the mechanisms by which these neurons select visual reference cues to anchor the head direction signals in relation to the environment.

The spatially selective responses of both hippocampal cells and HD cells are strongly influenced by landmark cues. In recordings in which rats forage for food in cylindrical enclosures, rotation of a contrasted card along the wall induces similar rotations of firing fields and of preferred directions (Muller et al., 1987; Taube et al., 1990; Taube, 1995; Zugaro et al., 2000). In simultaneous recordings, both types of cells respond coherently (Knierim et al., 1995).

\footnotetext{
Received Feb. 15, 2001; revised April 19, 2001; accepted April 23, 2001.

This work was supported by the Centre National de la Recherche ScientifiqueNational Science Foundation cooperation program, Centre National d'Etudes Spatiales, Cogniseine, Groupement d'Intérêt Scientifique. M.B.Z. received a grant from the Fondation pour la Recherche Médicale. We thank F. Maloumian for illustrations, P. Bernard, E. Camand, and A. Durand for help with experiments and data analysis, M.-A. Thomas and S. Doutremer for histology, and A. Treffel and M. Ehrette for the construction of the behavioral apparatus.

Correspondence should be addressed to S. I. Wiener, CNRS, Collège de France LPPA, 11 place Marcelin Berthelot, 75231 Paris CEDEX 05, France. E-mail: sidney.wiener@college-de-france.fr.

Copyright (C) 2001 Society for Neuroscience $\quad 0270-6474 / 01 / 210001-05 \$ 15.00 / 0$
}

was rotated. (The rat was disoriented in total darkness between sessions). This was repeated in the presence or absence of a cylinder enclosing the platform. When the enclosure was present, the preferred directions of all 30 cells recorded shifted by the same angle as the objects. In the absence of the enclosure, the preferred directions did not follow the objects, remaining fixed relative to the room. These results provide a possible neurophysiological basis for observations from psychophysical experiments in humans that background, rather than foreground, cues are preferentially used for spatial orientation.

Key words: foreground; background; landmark; spatial orientation; place cells; navigation

However, in experiments in which proximal and distal cues are displaced independently to present conflicting referents, place cells show a variety of responses: their firing fields stay fixed relative to either the distal cues, or the proximal cues, or the room, whereas other cells simply stop discharging (O'Keefe and Speakman, 1987; Wiener et al., 1995; Gothard et al., 1996; Tanila et al., 1997).

Cressant et al. (1997) compared the responses of hippocampal place cells before and after rotation of a group of objects that were always maintained in the same relative configuration. The firing fields rotated together with the objects when these were positioned in front of the wall of the enclosure. However, when the group of objects was placed near the center of the enclosure, then rotated, the firing fields did not follow. To account for this difference, the authors proposed that the centrally placed objects are ignored because the views of the configuration change too dramatically as the rat moves around in the cylinder. For example, an object can be seen either to the right or to the left of another object, depending on the position of the rat. This unreliability

This article is published in The Journal of Neuroscience, Rapid Communications Section, which publishes brief, peerreviewed papers online, not in print. Rapid Communications are posted online approximately one month earlier than they would appear if printed. They are listed in the Table of Contents of the next open issue of JNeurosci. Cite this article as: JNeurosci, 2001, 21:RC154 (1-5). The publication date is the date of posting online at www.jneurosci.org.

http://www.jneurosci.org/cgi/content/full/5390 
would render the cue configuration too complex to serve as a landmark.

To further characterize the respective roles of foreground and background visual cues for spatial orientation, here we examined head direction cell responses to rotations of a configuration of objects. However, instead of changing the eccentricity of the objects on the platform, we changed their relative depth with respect to the background, by testing the responses of the HD cells in the presence and absence of a cylindrical enclosure.

\section{MATERIALS AND METHODS}

The recording and analysis protocols are described in detail in Zugaro et al. (2000) and are summarized briefly here.

Electrode implantation. Three male Long-Evans rats (200-250 gm; CERJ, Le Genest-St-Isle, France) were tranquilized with xylazine, then deeply anesthetized with pentobarbital $(40 \mathrm{mg} / \mathrm{kg})$. The electrode bundles of eight formvar-coated nichrome wire electrodes (diameter, $25 \mu \mathrm{m}$; impedance, $200-800 \mathrm{k} \Omega$ ) were implanted above the anterodorsal nucleus of the thalamus (anteroposterior, $-1.6 \mathrm{~mm}$; mediolateral, $\pm 1.2 \mathrm{~mm}$ relative to bregma, $3.8 \mathrm{~mm}$ ventral to brain surface). Each bundle had been inserted in a 30 gauge stainless steel cannula and mounted on an advanceable connector assembly (Wiener, 1993). The descender assembly was permanently fixed with dental acrylic and tiny skull screws. Electrodes were gradually lowered until discriminable single-unit activity was detected. All protocols were in accord with institutional, national, and international standards and regulations.

Data acquisition. During the recording sessions, electrode signals passed through field effect transistors and were differentially amplified $(10,000 \times)$ and filtered $(300 \mathrm{~Hz}$ to $5 \mathrm{kHz}$, notch at $50 \mathrm{~Hz})$. The signal was then acquired on a DataWave Discovery system (Longmont, CO). Two small infrared light-emitting diodes (10 $\mathrm{cm}$ separation) mounted above the headstage were detected by a video camera. To determine the preferred direction of an HD cell, head angles were computed from smoothed corrected position samples, and directional response curves were fit with a pseudo-Gaussian function according to the method of Zugaro et al. (2000).

Experimental setup. The $3 \times 3 \times 3 \mathrm{~m}$ square recording chamber was surrounded by black curtains $(3 \mathrm{~m}$ high) suspended from the ceiling along the four walls. The folds of the curtains were rather irregular $(15-30 \mathrm{~cm}$ wide). The ceiling was also covered by a black curtain. Illumination was provided by a $40 \mathrm{~W}$ overhead lamp on the ceiling that diff used light evenly within the cylinder. All electronic instruments and computers were situated outside of the curtains, and the entire experimental room was phonically isolated from the rest of the building (Fig. 1).

During the experiments the rats moved freely on an elevated platform (75 $\mathrm{cm}$ above the floor), measuring $90 \times 90 \mathrm{~cm}$ in earlier experiments and $76 \mathrm{~cm}$ diameter in later experiments. Each cell was recorded under two experimental conditions.

Proximal background condition. Here, a black cylindrical enclosure (60 $\mathrm{cm}$ high, $76 \mathrm{~cm}$ in diameter) was placed on the platform (Fig. 1A). This prevented the rat from viewing the curtains along the walls of the room. Three objects were placed in a triangular spatial configuration along the inner wall of the enclosure: a black cone ( $26 \mathrm{~cm}$ high, $22 \mathrm{~cm}$ in diameter), a cylinder covered with light brown paper $(26 \mathrm{~cm}$ high, $10 \mathrm{~cm}$ in diameter), and a building brick $(22 \times 11 \times 5 \mathrm{~cm})$. The spatial configuration of the objects relative to one another never changed in the experiments, and each experiment started with the objects in the same configuration relative to the room.

Distal background condition. Here, the black cylindrical enclosure was absent, and the more distant surrounding curtains provided a background for the objects (Fig. $1 B$ ). The objects occupied the same placements as in the proximal background condition. The displacements of the rats were restricted to the platform area because this was elevated above the floor.

Behavioral task. The proximal background condition was always tested first. The experimental procedure was similar in both conditions. First, to determine the preferred direction of the cells, the rat was allowed to move freely within the arena for at least $5 \mathrm{~min}$, foraging for small food pellets ( $5 \mathrm{mg}$ chocolate sprinkles) thrown onto the platform at pseudorandom locations (Muller et al., 1987). The rat was then removed from the arena and secluded in a small opaque container. The objects were rotated by $120^{\circ}$ clockwise or counterclockwise (Fig. $2 A, B$ ), and the floor paper was changed. To disorient the rat, all lights were turned off
A

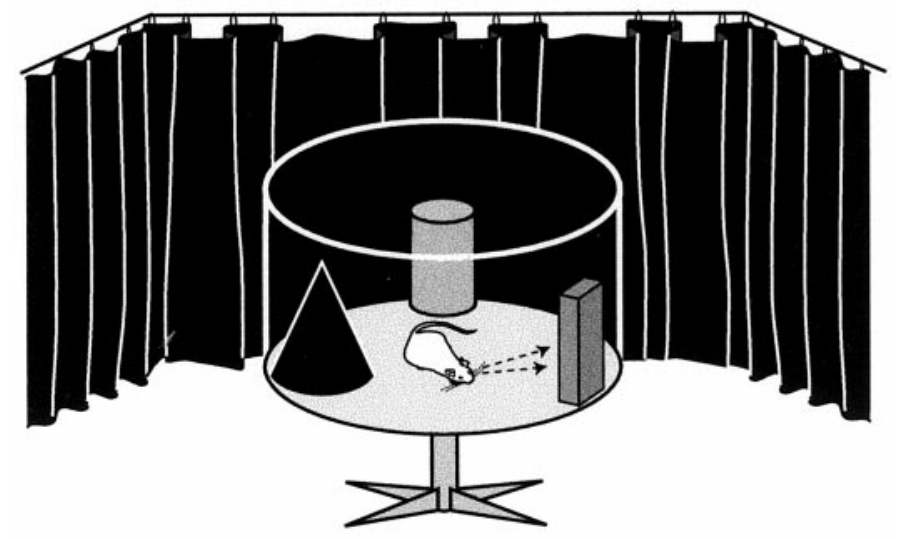

B

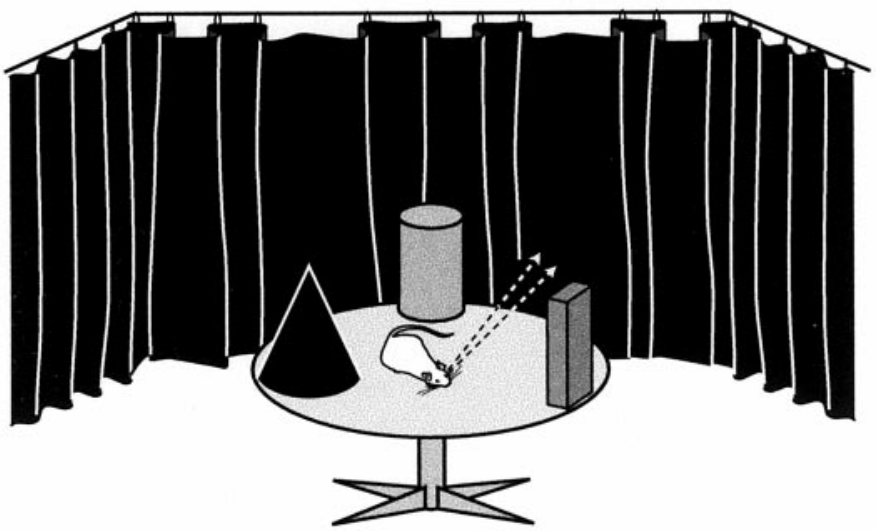

Figure 1. The experimental setup. The elevated platform was surrounded by black curtains hanging along the four walls. A cone, a cylinder, and a building brick placed on the platform served as orienting cues. $A$, In the proximal background condition, a black cylindrical enclosure was placed on the platform, restricting view and movements of the rats. $B$, In the distal background condition, the enclosure was absent. Thus, the surrounding black curtains were a more distal backdrop for the threedimensional objects.

(including the instrument lamps), and an experimenter rotated the opaque container in an erratic manner while wandering around the room for 1 or $2 \mathrm{~min}$. The rat was then replaced in the arena from a pseudorandomly selected orientation, the lights were turned back on, and a second 5 min recording session was started. This manipulation was performed for both the proximal and distal background conditions. In the distal background condition, when the enclosure was absent, an experimenter remained in the room with the rat, to throw food pellets onto the platform. As during the proximal background condition, the experimenter kept moving around the room to provide no stable reference information.

Histology. At the end of the experiments, the recording sites were marked by passing a small cathodal DC current ( $30 \mu \mathrm{A}, 10 \mathrm{sec})$ through one of the recording electrodes. The rat was then anesthetized with a lethal dose of pentobarbital. Intracardial perfusion with saline was followed by $10 \%$ formalin-saline. Histological sections were stained with cresyl violet. Recording sites were determined by detecting the small lesion and the track created by the cannula. In all cases, analyses of these data indicated that the recording sites were indeed in the anterodorsal nucleus of the thalamus. 


\section{Proximal Background Condition}

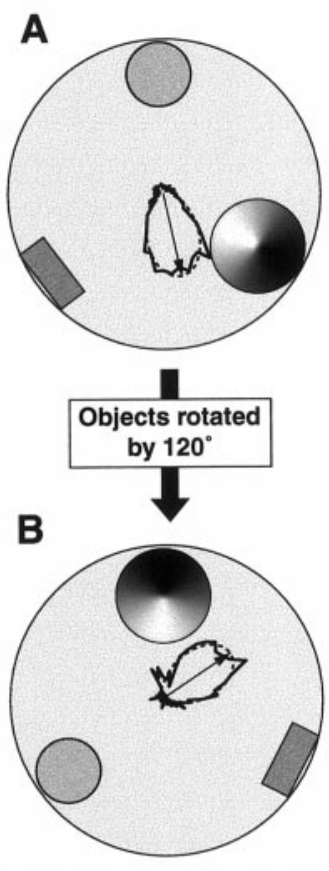

\section{Distal Background Condition}

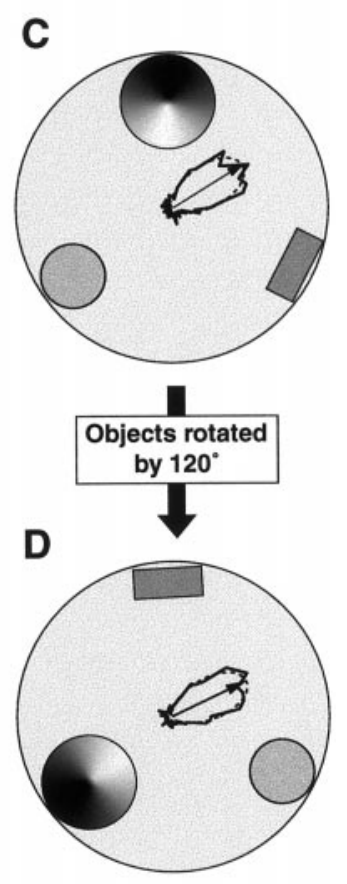

Figure 2. Directional response curves of a typical HD cell recorded while the objects were at their initial positions $(A, C)$ and after they were rotated by $120^{\circ}(B, D)$ while the rat was secluded in darkness. The directional response curves (continuous curves) are plotted along with their Gaussian-like fits (dashed curves). $A, B$, In the presence of the enclosure, the preferred direction of this neuron shifted by $112^{\circ}$. C, $D$, When the enclosure was absent, the preferred direction of the cell remained virtually unchanged after the objects were rotated by $120^{\circ}$.

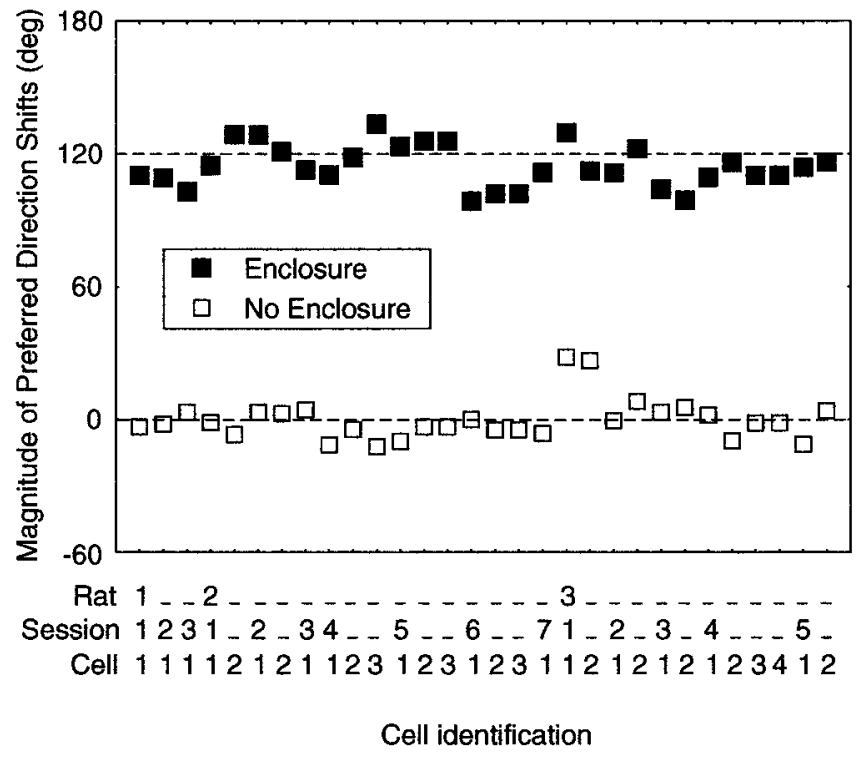

Figure 3. Responses of the cells after rotation of the objects by $120^{\circ}$. Dashed lines indicate responses anchored to the objects $\left(120^{\circ}\right)$ or to the room $\left(0^{\circ}\right)$ (shifts in preferred directions are measured relative to the room). In the presence of the enclosure, the preferred directions followed the objects (filled squares). When the enclosure was absent, the preferred directions remained fixed relative to the room (open squares). All object rotations were counterclockwise, except for rat 1 -session 2 and rat 2-session 1 . On the $x$-axis, repeated recordings from the same rat or session are indicated by dashes.

\section{RESULTS}

\section{Proximal background condition}

Figure 2 shows the typical response of an anterodorsal thalamic HD cell recorded before $(A)$ and after $(B)$ the objects were rotated $120^{\circ}$ while the cylindrical enclosure was present, providing a proximal background. The preferred direction of this cell shifted (relative to the room) $109^{\circ}$ from the first to the second trial, anchored to the $120^{\circ}$ rotation of the objects. Similarly, in all 30 of the HD cells recorded in the three rats, the preferred directions shifted by the same angle as the objects (Fig. 3, filled squares). The average magnitude of the shift was $115 \pm 10^{\circ}(\mathrm{SD}$; range, $\left.99-134^{\circ}\right)$.

\section{Distal background condition}

Figure 2 shows the responses of the same HD cell recorded before $(C)$ and after $(D)$ the objects were rotated by $120^{\circ}$ in the absence of the proximal background provided by the cylindrical enclosure. The preferred direction of this cell shifted by only $-6^{\circ}$ between the two trials, despite the rotation of the objects. Similarly, negligible shifts in preferred directions were observed in the population of HD cells recorded in the three rats (Fig. 3, open squares). The average magnitude of the shifts was only $6 \pm 7^{\circ}(\mathrm{SD}$; range, $0-28^{\circ}$ ).

One possible explanation for the lack of influence of the objects on the preferred directions in the distal background condition was that they may have no longer been salient. For example, the four curtains may have distracted the rats from attending to the objects. The curtains could have been salient because of contrasts in the folds. To test for evidence of this, the time that the rats spent near the objects after object rotations was compared between the proximal and distal background conditions. This was measured as the time spent in the vicinity $(\leq 6 \mathrm{~cm})$ of the objects. On average, 
the rats spent $21 \pm 6 \%$ (SD; range, $15-32 \%$ ) of the time near the objects when the enclosure was present and $20 \pm 4 \%$ (range, 13-30\%) when the enclosure was absent. There was no significant difference in the two conditions (Wilcoxon matched pairs test, $N=15$; NS) Thus, the objects maintained their saliency in the distal background condition.

Other response properties of the HD cells were unaffected by the removal of the enclosure: comparisons between the sessions preceding and after removal of the enclosure showed no significant difference in peak firing rates or angular response ranges (Wilcoxon matched pairs tests, $N=30$; NS)

\section{DISCUSSION}

The preferred directions of the HD cells reoriented after rotations of a group of objects, but only when the objects were close to the visual background, the cylindrical enclosure. There are several possible explanations for the reduced efficacy of the objects when the cylindrical enclosure was absent and the distant curtains were then in the background: (1) as suggested by Cressant et al. (1997), the configuration of the objects was rejected as an unreliable reference because the rat could view them from different perspectives, (2) in the distal background condition, the curtains proved to be a larger and more salient reference landmark than the objects, (3) in the distal background condition, the geometric characteristics of the square room influenced the responses of the cells, and (4) in both conditions the most distal cues were used as reference landmarks for the head direction cells; these corresponded to the objects in the proximal background condition and to the curtains in the distal background condition. These will be discussed in the following sections.

\section{Was the object configuration rejected as an unreliable reference in the distal background condition because it provided ambiguous orienting information?}

In our proximal background condition [and the experiment with the objects placed next to the wall of the enclosure of Cressant et al. (1997)], the rats explored the narrow space between the objects and the cylindrical enclosure. This could also have provided an ambiguous view of the spatial configuration of the objects but did not reduce the influence of the objects. Furthermore, in our experiments when the cylindrical enclosure was absent, the limits of the platform prevented the rats from having access to mirror image views of the relative positions of the objects, as was the case for the centrally placed objects of Cressant et al. (1997). Nonetheless the objects no longer influenced the preferred directions of the head direction cells. In addition, the preferred directions appeared to be established within seconds after the rat is placed on the platform (our unpublished observations; also see Zugaro et al., 2000), before it explored a substantial part of the arena. Finally, in the Cressant et al. (1997) study, the firing fields often rotated by arbitrary angles after the centrally placed objects were rotated. This indicates that the firing fields were not anchored to any particular cue in the environment. In contrast, here the preferred directions were always maintained fixed relative to the room after rotations of the objects in the distal background condition. Thus, it appears that the preferred directions not only were independent from the orientation of the group of objects but were controlled by other cues in the more distant background (possibly contrasts in the curtains).

\section{Were the objects too small and insufficiently salient in the distal background condition?}

After the cylindrical enclosure was removed, the curtains provided a large and contrasted background. But the cylindrical enclosure, because of its height and proximity, subtended a larger visual angle than the curtains did in the distal background condition. The curtains, with their contrasted folds, were likely to have been salient. However, as shown above, the rats frequented the rotated objects equally in the presence and absence of the cylindrical enclosure. Thus, the objects were likely to have remained salient even when they were no longer effective in controlling the preferred directions.

\section{Did the geometry of the square room control the preferred directions in the distal background condition?}

Although rats can ignore landmark cues and instead navigate based on the geometry of the environment (Cheng, 1986; Margules and Gallistel, 1988), it is unlikely that the preferred directions of the HD cells were influenced by the geometry of the square room when the cylindrical enclosure was absent. First, the room geometry has been shown to influence orienting behavior in asymmetric (rectangular) environments. Here, there were no evident differences in the metrics of the four corners or the four walls. If the geometry of the square room was a controlling factor, one would predict that the preferred directions would have rotated by multiples of $90^{\circ}$ because of its symmetry. However, in the distal background condition, the HD cells always retained the same preferred directions after the objects were rotated.

\section{Were the most distal cues used as reference landmarks for anchoring preferred directions in both conditions?}

Another possible explanation for the difference in efficacy of the very same objects in the two experimental conditions is that their relative distance to the background changed. Relative depth in the visual field could be detected on the basis of several different stimulus attributes including occlusion (objects blocked by others are more distant), parallax (during active displacements more distant objects appear to move less), texture contrast, shadows, vergence, etc. This criterion would be functionally relevant because stimuli that are furthest in the background remain more stable as the animal moves around and thus would be more reliable as landmarks.

Brain systems for detecting optic field flow could provide this sensitivity to the head direction system because, for example, the optokinetic system is more sensitive to optic flow at low, rather than high velocities (Hess et al., 1985). An anatomical pathway that could convey optokinetic information to head direction cells passes via the vestibular nuclei to the dorsal lateral tegmental nucleus of Güddens, then the lateral mammillary nuclei before arriving at the anterodorsal thalamic nucleus. As the rat makes displacements, the more distant objects provide the slowest optic field flow velocities. This would help the head direction system select those parts of the visual field providing the most stable reference points and also help update preferred directions after self-movements.

The hypothesis that the objects no longer controlled the preferred directions of the HD cells because they no longer were background cues is coherent with the conclusions of a psychophysical study comparing the relative importance of visual cues in the foreground versus the background in controlling vection (the visu- 
ally induced sensation of motion) in human subjects. Brandt et al. (1975) showed that the apparent self motion produced by moving contrasts was strongly reduced when stationary contrasts were present in the background. The authors concluded that “... spatial orientation relies mainly on the information from the seen periphery ...".

It remains to be determined precisely what stimulus attributes of background cues are most effective at driving head direction cells. Nonetheless, our proposed explanation is also applicable to the results from place cell recordings (Cressant et al., 1997). This is consistent with the notion that the head direction signal feeds into the hippocampus and that hippocampal firing fields and HD cell preferred directions are updated in a coherent manner (Knierim et al., 1995).

\section{REFERENCES}

Brandt T, Wist ER, Dichgans J (1975) Foreground and background in dynamic spatial orientation. Percept Psychophys 17:497-503.

Cheng K (1986) A purely geometric module in the rat's spatial representation. Cognition 23:149-178.

Cressant A, Muller RU, Poucet B (1997) Failure of centrally placed objects to control the firing fields of hippocampal place cells. J Neurosci 17:2531-2542.

Gothard KM, Skaggs WE, McNaughton BL (1996) Binding of hippocampal CA1 neural activity to multiple reference frames in a landmark-based navigation task. J Neurosci 16:823-835.

Hess BJ, Precht W, Reber A, Cazin L (1985) Horizontal optokinetic ocular nystagmus in the pigmented rat. Neuroscience 15:97-107.

Khabbaz A, Fee MS, Tsien JZ, Tank DW (2000) A compact convergingelectrode microdrive for recording head direction cells in mice. Soc Neurosci Abstr 26:984.

Knierim JJ, Kudrimoti H, McNaughton BL (1995) Hippocampal place fields, the internal compass, and the learning of landmark stability. J Neurosci 15:1648-1659.

Margules J, Gallistel CR (1988) Heading in the rat: determination by environmental shape. Anim Learn Behav 16:404-410.
McHugh TJ, Blum KI, Tsien JZ, Tonegawa S, Wilson MA (1996) Impaired hippocampal representation of space in CA1-specific NMDAR1 knockout mice. Cell 87:1339-1349.

Muller RU, Kubie JL, Ranck Jr JB (1987) Spatial firing patterns of hippocampal complex-spike cells in a fixed environment. J Neurosci 7:1935-1950.

O'Keefe J, Conway DH (1978) Hippocampal place units in the freely moving rat: why they fire where they fire. Exp Brain Res 31:573-590.

O'Keefe J, Speakman A (1987) Single unit activity in the rat hippocampus during a spatial memory task. Exp Brain Res 68:1-27.

Ono T, Tamura R, Nakamura K (1991) The hippocampus and space: are there "place neurons" in the monkey hippocampus? Hippocampus $1: 253-257$.

Ranck Jr JB (1984) Head-direction cells in the deep cell layers of dorsal presubiculum in freely moving rats. Soc Neurosci Abstr 10:599.

Robertson RG, Rolls ET, Georges-François P, Panzeri S (1999) Head direction cells in the primate pre-subiculum. Hippocampus 9:206-219.

Rotenberg A, Mayford M, Hawkins RD, Kandel ER, Muller RU (1996) Mice expressing activated CaMKII lack low frequency LTP and do not form stable place cells in the CA1 region of the hippocampus. Cell 87:1351-1361.

Tanila H, Shapiro M, Gallagher M, Eichenbaum H (1997) Brain aging: changes in the nature of information coding by the hippocampus. J Neurosci 17:5155-5166.

Taube JS (1995) Head direction cells recorded in the anterior thalamic nuclei of freely moving rats. J Neurosci 15:70-86.

Taube JS (1998) Head direction cells and the neurophysiological basis for a sense of direction. Prog Neurobiol 55:1-32.

Taube JS, Muller RU, Ranck Jr JB (1990) Head-direction cells recorded from the postsubiculum in freely moving rats. I. Description and quantitative analysis. J Neurosci 10:420-435.

Wiener SI (1993) Spatial and behavioral correlates of striatal neurons in rats performing a self-initiated navigation task. J Neurosci 13:3802-3817.

Wiener SI, Korshunov VA, Garcia R, Berthoz A (1995) Inertial, substratal and landmark cue control of hippocampal CA1 place cell activity. Eur J Neurosci 7:2206-2219.

Zugaro MB, Tabuchi E, Wiener SI (2000) Influence of conflicting visual, inertial and substratal cues on head direction cell activity. Exp Brain Res 133:198-208. 